\title{
Preemptive re-association scheme for beacon-enabled wireless personal area networks
}

Faisal Bashir Hussain ${ }^{1}$ and Jae-Young Pyun ${ }^{2 *}$

\begin{abstract}
Communication in wireless personal area networks (WPANs) is characterized as short range (up to few meters). In beacon-enabled cluster-tree-based WPANs, node mobility can result in several cell changes. Each cell change is detected after the connectivity with previous cell is broken. Moreover, end devices are unable to transmit or receive application data while performing time-consuming scanning and re-association procedures. In this scenario, frequent node mobility can degrade the performance of WPAN. In existing literature, research efforts are made to improve connectivity and re-association procedure individually, but due to their contradictory assumptions, they cannot be used together. In this work, we modify the re-association procedure of IEEE 802.15.4 to decrease both re-association frequency and latency for a beacon-enabled cluster-tree-based WPAN with mobile end devices and coordinators.
\end{abstract}

Keywords: WPAN; Association; Coordinator discovery; Passive scan

\section{Introduction}

IEEE 802.15.4-based wireless personal area networks (WPANs) have emerged as the most popular choice for monitoring and control-oriented sensory applications. This is because of their unattended and self-configuring mode of operation. Also, specialized network topologies like cluster-tree can provide larger coverage with reduced energy consumption [1]. Supporting mobility of devices in WPANs is vital for growing application demands. Mobility of devices is imminent in many WPAN applications such as habitat monitoring [2], health care systems [3], emergency scenarios [4], and inventory management [5].

Mobility of devices in short-range WPANs seriously hinders the performance due to frequent loss of connectivity $[6,7]$. In beacon-enabled cluster-tree-based WPANs, when a device is unable to receive beacons from its coordinators, its connectivity is lost. Therefore, re-association process is triggered. Node re-association defines the procedures for discovering coordinator(s) and joining with coordinator. This re-association process comprises of orphan scan, passive scan, and later association message

\footnotetext{
*Correspondence: jypyun@chosun.ac.kr

2 Department of Information and Communication Engineering, Chosun University, Gwangju 501-759, South Korea

Full list of author information is available at the end of the article
}

exchange. The orphan scan is used to re-synchronize with parent coordinator, if the node loses connection with the coordinator. Passive scan is used to find a new coordinator. Association-related messages are exchanged between the device and the new coordinator to obtain network address and synchronization. Before re-association process is completed, the associating device is unable to transmit and receive application data.

The frequency of re-associations depends on node speed and network topology. At higher node speeds and sparse network deployment, nodes can frequently lose connectivity, resulting into higher re-association frequency. In order to reduce the frequency of re-association in mobile environment, coordinators that can provide longer connectivity are better suited for association. On the other hand, the latency of re-association processes primarily depends on coordinator discovery during orphan and passive scans. The channel scanning durations are defined based on the beacon order (BO) of the coordinators. The longer is the $\mathrm{BO}$, the higher is the latency of coordinator discovery because of long beacon interval.

In this paper, we investigate the problems of re-association in beacon-enabled cluster-tree-based WPANs. Then, a preemptive re-association (PRA) scheme is proposed for decreasing both frequency and latency of re-association

\section{焦 Springer}


process. Contrary to IEEE 802.15.4, PRA attempts to detect loss of connectivity preemptively, i.e., before the actual loss occurs. Moreover, PRA performs fast reassociation procedure and selects coordinators which can provide longer connectivity during re-association of mobile devices. In the case of coordinator discovery in higher $\mathrm{BO}$ networks, PRA also attempts to decrease the beacon interval of coordinators which are in close vicinity of mobile end devices. This paper is organized as follows. Section 2 outlines existing research efforts on node association in WPANs. Sections 3 and 4 present the network model and operation of the proposed PRA scheme, respectively. Simulation results are analyzed in Section 5, whereas the last section concludes the paper.

\section{Related work}

In the existing literature, the problem of re-association has been referred under mobility management [8], increasing connectivity [9], coordinator discovery $[10,11]$, and association schemes [12-14]. However, re-association process for reducing both re-association frequency and latency in mobile environments has not been studied to the best of our knowledge.

A mobility management scheme for cluster-tree-based WPANs is presented in [8]. It uses a speculative algorithm for node association based on link quality indicator (LQI) value of received coordinator beacons. When a cell change is expected, it triggers active scan to find new coordinator. In order to increase sensor node connectivity time with coordinator, [9] proposes the use of time-stamped beacons with highest LQI for coordinator selection. Nevertheless, this scheme does not provide any contributions for decreasing the overall association time.

Assessment of passive discovery in IEEE 802.15.4 body area sensor networks for targeted and untargeted scenarios is presented in [10]. This work concludes that in targeted discovery of pre-defined coordinator, increasing the success rate of discovery significantly increases the cost of discovery. In untargeted discovery of any available coordinator, it is preferable to stay longer on one channel. Also, if $\mathrm{BO}$ of coordinators is high, they are only rarely discovered. In [11], an asynchronous neighbor discovery protocol called Disco is presented. A pair of prime numbers is selected by each node in a way that the sum of their reciprocal is equal to the desired duty cycle. Whenever a node counter is a multiple of either prime number, the node becomes active and starts to transmit or receive. This work ensures that the discovery of coordinator is in bounded time. However, their work is not based on IEEE 802.15.4.

Collaborative node association [12] uses location information of the mobile sensors. Each node is assumed to be position aware. When sensors are mobile and they move from one router to another, two adjacent routers cooperate to complete the association procedure. Simple association process (SAP) [13] is an enhancement to IEEE 802.15.4. It is designed to reduce redundant primitives and decrease association delay. But, it does not address the issue of node mobility and is proposed for network initialization period. Fast association mechanism [14] is proposed for real-time WPAN applications. Delay caused by scanning multiple channels is reduced in [14] because the scanning process is terminated as soon as any beacon is received. This work can be applied to partially decrease re-association latency but it can not reduce re-association frequency. In our previous work [15], a coordinator-assisted passive discovery scheme for beacon-enabled IEEE 802.15.4 networks is presented. This work focuses on reducing the latency of handover by preemptively detecting need for handover using LQI. However, this work does not consider on reducing handover frequency and also assumes a partially mobile network, with mobility of end devices only.

\section{Network model}

In this section, our network model, definitions, and assumptions are explained. We consider a beaconenabled and cluster-tree-based WPAN. PAN coordinator is assumed to be static while fully functional devices (FFDs) and reduced functional devices (RFDs) can be mobile. Low mobility is considered with random movement of devices. All coordinators within the network transmit beacons. Moreover, they can dynamically adjust their beacon intervals for optimizing the duty cycle of PAN devices using BO selection algorithms $[16,17]$. The terms of coordinator and cluster head are interchangeably used in this paper, referring to beacon-enabled FFDs. Furthermore, it is assumed that FFDs can detect their acceleration to categorize themselves as mobile or nonmobile.

The basic network design of clustered-tree-based topology is shown in Figure 1. Node 0 is the PAN coordinator whereas nodes 8,9 , and 11 are the end devices. The rest of the nodes are cluster heads. A cluster is a small section of the bigger network that has its own FFD acting as a cluster head. The large circle corresponds to the coverage (radio range) of cluster head 3 . Node 8 moves from location $\left(x_{1}\right.$, $\left.y_{1}\right)$ to $\left(x_{2}, y_{2}\right)$ at any instance of time $t_{1}$. It further moves away to location $\left(x_{3}, y_{3}\right)$ at time $t_{2}$. When node 8 leaves the radio range of cluster head 3 at $\left(x_{2}, y_{2}\right)$, non-availability of coordinator is detected by the loss of successive beacons at the end device. In this work, we assume that a node may not receive the coordinator's beacon due to mobility of the coordinator or its own mobility. Loss of connectivity triggers orphan scan, passive scan, and later association messages are exchanged, as shown in Figure 2. Following observations are made during this re-association of mobile end device after cluster change. 


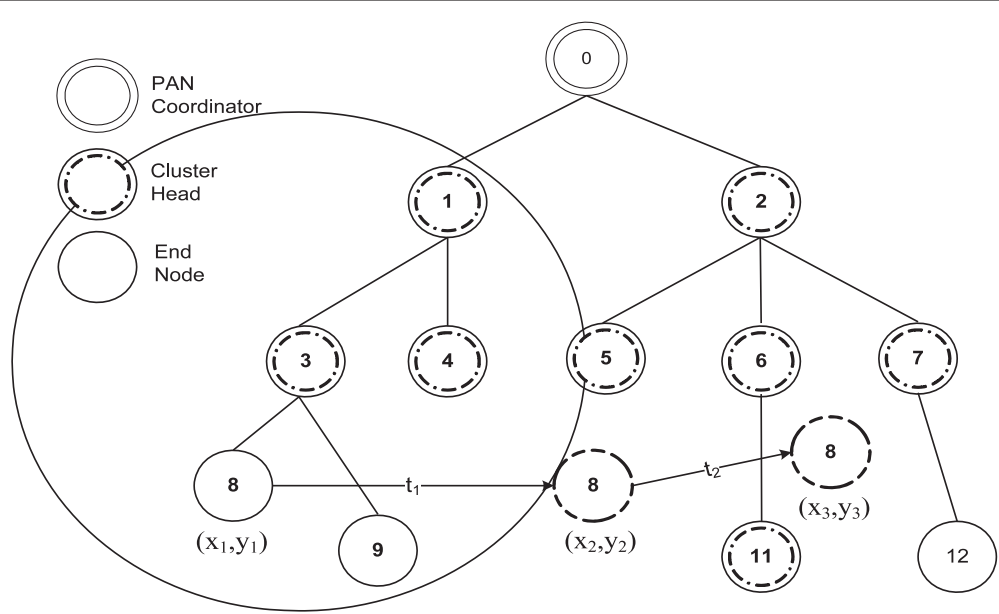

Figure 1 Network model for cluster-tree topology.

- The re-association is triggered after connectivity with the previous coordinator is lost. Hence, the cluster handover uses a break-before-make scheme for re-association that increases latency of re-association as compared to make-before-break scheme.

- The re-association process uses fixed length orphan scan on each physical channel to discover the parent coordinator and re-synchronize with it. If orphan scan fails, passive scan is then used to find new coordinator. Because mobile node cannot re-synchronize with parent coordinator after cell change, performing orphan scan is unnecessary and time-consuming.

- The passive scan duration depends on network BO and number of IEEE 802.15.4 physical channels. If BO of network is high then re-association is difficult to achieve because of longer beacon interval length, thus significantly raising the re-association latency.

- After passive scan, devices can find multiple coordinators for possible re-association. For example, in Figure 1, when node 8 performs passive scan for re-association at $\left(x_{2}, y_{2}\right)$ coordinates, it discovers coordinators 4, 5, 6, and 10. Appropriate coordinator selection is prerequisite for increasing connectivity and decreasing re-association frequency. However, IEEE 802.15.4 does not specify any mechanism for appropriate coordinator selection and only suggests the use of LQI.

From the above observations, it is evident that performance of WPANs under mobility of devices can be enhanced by decreasing both latency and frequency of

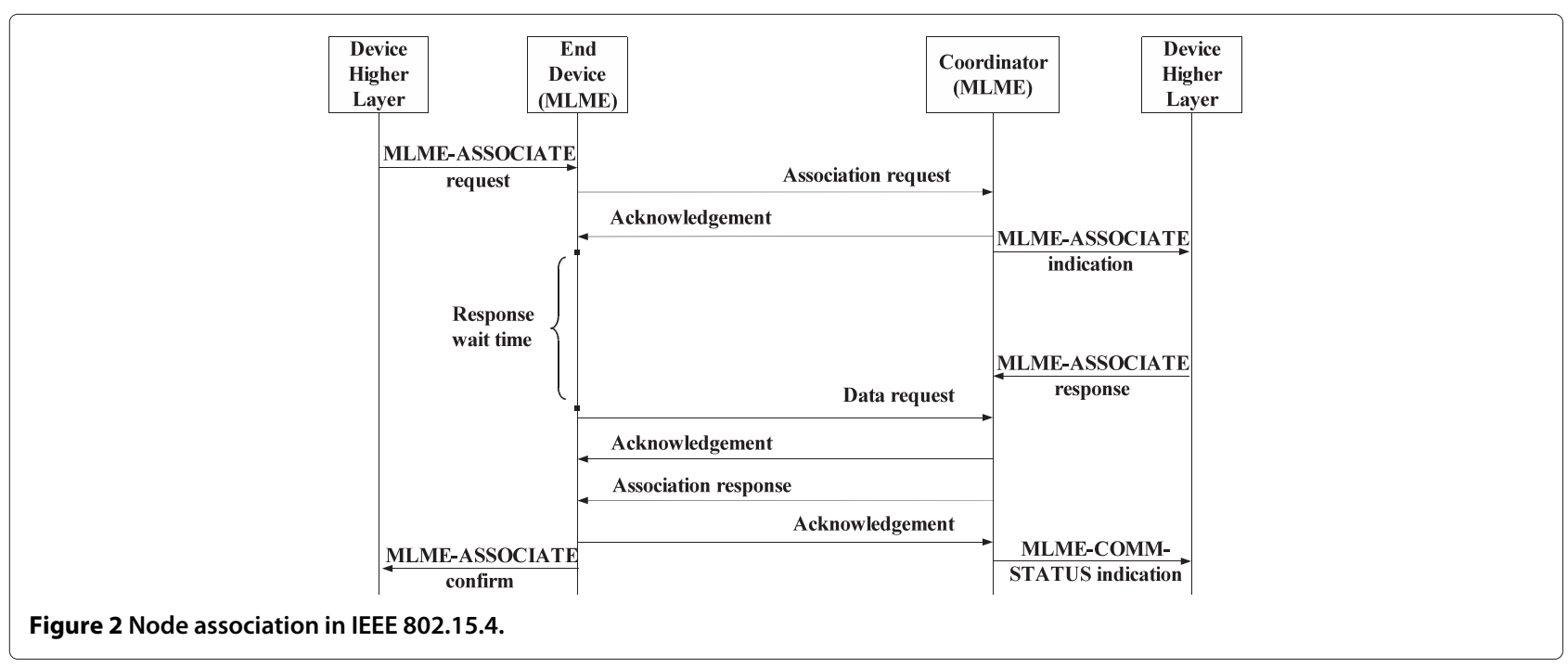


re-association. In this work, the latency of re-association during cell change is decreased using the following modifications. Firstly, make-before-break approach is used that reduces the latency of link down detection after cluster change. Secondly, orphan scan is not used because in a make-before-break approach, the end device is already connected to parent coordinator while trying to find new coordinator. Thirdly, passive scan is performed on current channel to decrease the scanning time. Lastly, for high BO networks, beacon order of neighboring coordinators is decreased when weak connectivity is detected by parent coordinator with any of its member device. This helps the weakly connected member device to swiftly select the new coordinator. On the other hand, to decrease the frequency of handover, a new coordinator selection criterion is proposed. It takes into account the quality of link between the devices and mobility of coordinator. As a result, connectivity can be enhanced by selecting both stronger and less mobile links.

\section{Preemptive re-association scheme}

The operation of PRA scheme consists of preemptive re-association decision (PRD) and re-association procedure (RAP). PRD is taken by the coordinator to find which member device has low connectivity and should trigger its RAP. In order to ensure that devices have makebefore-break connectivity, PRD attempts to find weakly connected nodes based on LQI before the link is broken. RAP illustrates the sequence of steps required to associate with a new coordinator that can give longer connectivity. In the remaining of this section, both PRD and RAP are explained in length.

\subsection{Preemptive re-association decision}

LQI is an important metric available in IEEE 802.15.4 for detection of link quality between two devices. Makebefore-break approach requires either devices or coordinator to monitor the LQI for preemptively triggering the re-association during the cluster change. Member devices can use the LQI of parent coordinator beacons to take re-association decision $[8,9]$. However, the frequency of beacons can be low or high depending on $\mathrm{BO}$ of coordinator. At higher $\mathrm{BO}$, beacons are received after longer intervals resulting in delayed detection of link quality. In PRD, the coordinator monitors the link quality of end devices. This moves the computational overhead from end devices to coordinator and provides more accurate link detection for event reporting nodes.

LQI of received packets is calculated as a minimum function of energy detection (ED) and signal-to-noise ratio (SNR). LQI has a value between 127 and 255, where 127 is the minimum and 255 is the maximum link quality. If successive readings of LQI from a member node are dropping, then the coordinator communicates with end device to discover new coordinator for re-association. Link quality can degrade due to increase in distance, interference, and malfunctioning of devices. As a result, observed LQI values can fluctuate at any instance of time and can be proportional to distance in the next instance of time. Therefore, PRD decision cannot be made on per packet basis, instead successive LQI reading are considered. The flow chart of PRD including link quality detection is shown in Figure 3. When continuous decrease in link quality of member node is below LQI threshold, the member node is added in the weak node list of coordinator. At the end of each beacon interval, the coordinator transmits WEAK_NODE_LIST in the beacon.

\subsection{Re-association procedure}

Devices perform preemptive re-association, when their link quality is low with their parent coordinator. Low connectivity is communicated by the coordinator through beacon containing the weakly connected node list. The RAP flow chart is shown in Figure 4. Contrary to IEEE 802.15.4, passive scan is initiated first to find any possible coordinator(s), instead of re-synchronization attempt using orphan scan. Orphan scan is only invoked if connectivity with parent coordinator is lost, that is detected by beacon loss. Since RAP is invoked before the connectivity with parent coordinator is lost, orphan scan is not required.

A number of coordinators can be found during the passive scan, depending on coordinator density and BO. Appropriate coordinator selection is trivial for increasing connectivity between the devices. During RAP, coordinator having the highest weight is selected for association. Coordinator weight $(\mathrm{CW})$ is based on LQI of the received beacon and mobility factor (MF) of the coordinator. CW is determined as follows:

$$
\mathrm{CW}=\alpha \times\left(\frac{\mathrm{LQI}_{\text {beacon }}}{\mathrm{LQI}_{\min }}-1\right)+\beta \times(1-\mathrm{MF})
$$

where, $127 \leq$ LQI $_{\text {beacon }} \leq 254, \mathrm{LQI}_{\min }=127,0 \leq \mathrm{MF} \leq 1$, and $1 \leq \alpha, \beta \leq 2$. MF is the measure of coordinator mobility in recent past. If during the beacon interval, the coordinator moves, then its MF is 1 otherwise 0 . Simple moving average of MF during the last ten intervals is transmitted in the beacon frame. CW rises with increase in link quality and decrease in mobility of coordinator. In Equation $1, \alpha$ and $\beta$ are the weights for link quality and MF, respectively. Selection of coordinator can be determined according to network dynamics, using appropriate values for $\alpha$ and $\beta$. In the case of high mobility of coordinators, more weight can be assigned to MF. On the other hand, in the case of low mobility of coordinators, more weight can be assigned to LQI. 


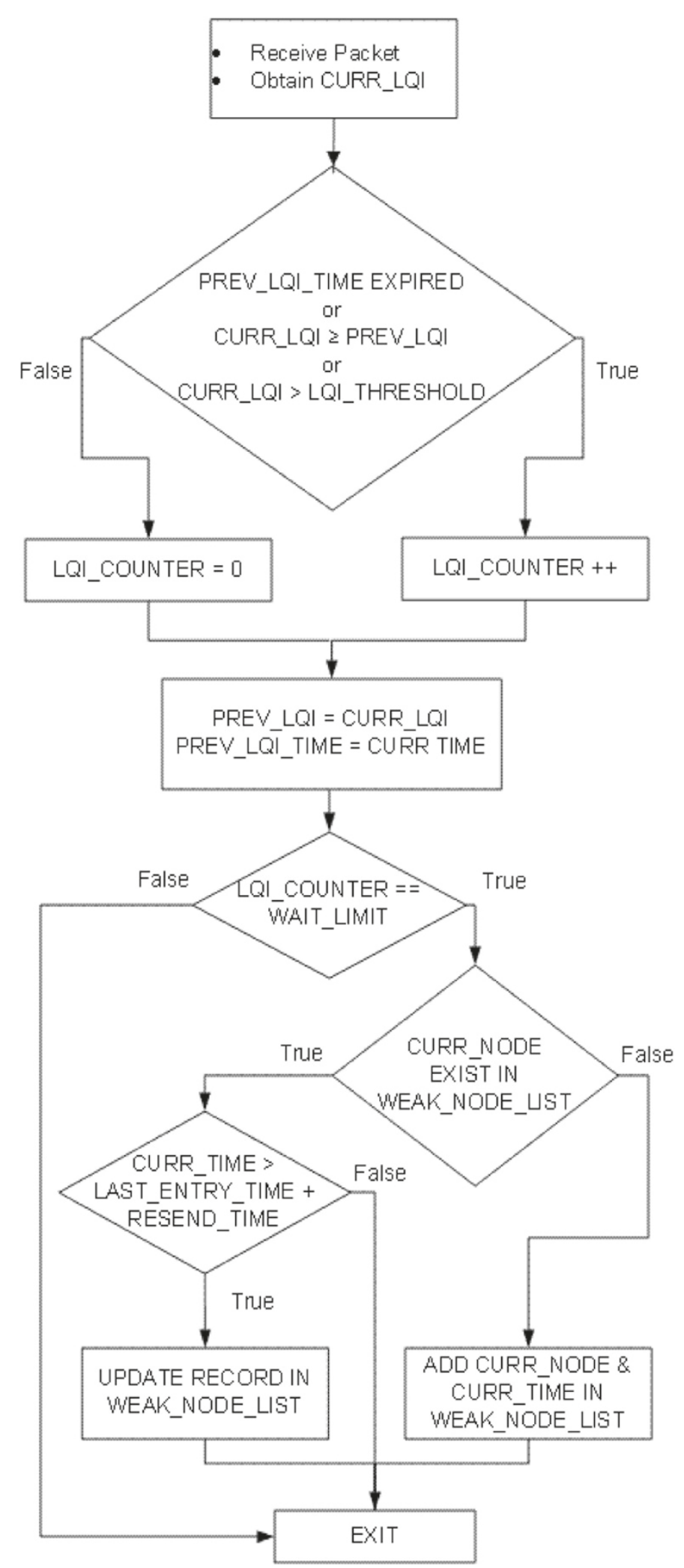

Figure 3 Flow chart: preemptive re-association decision at coordinator. 


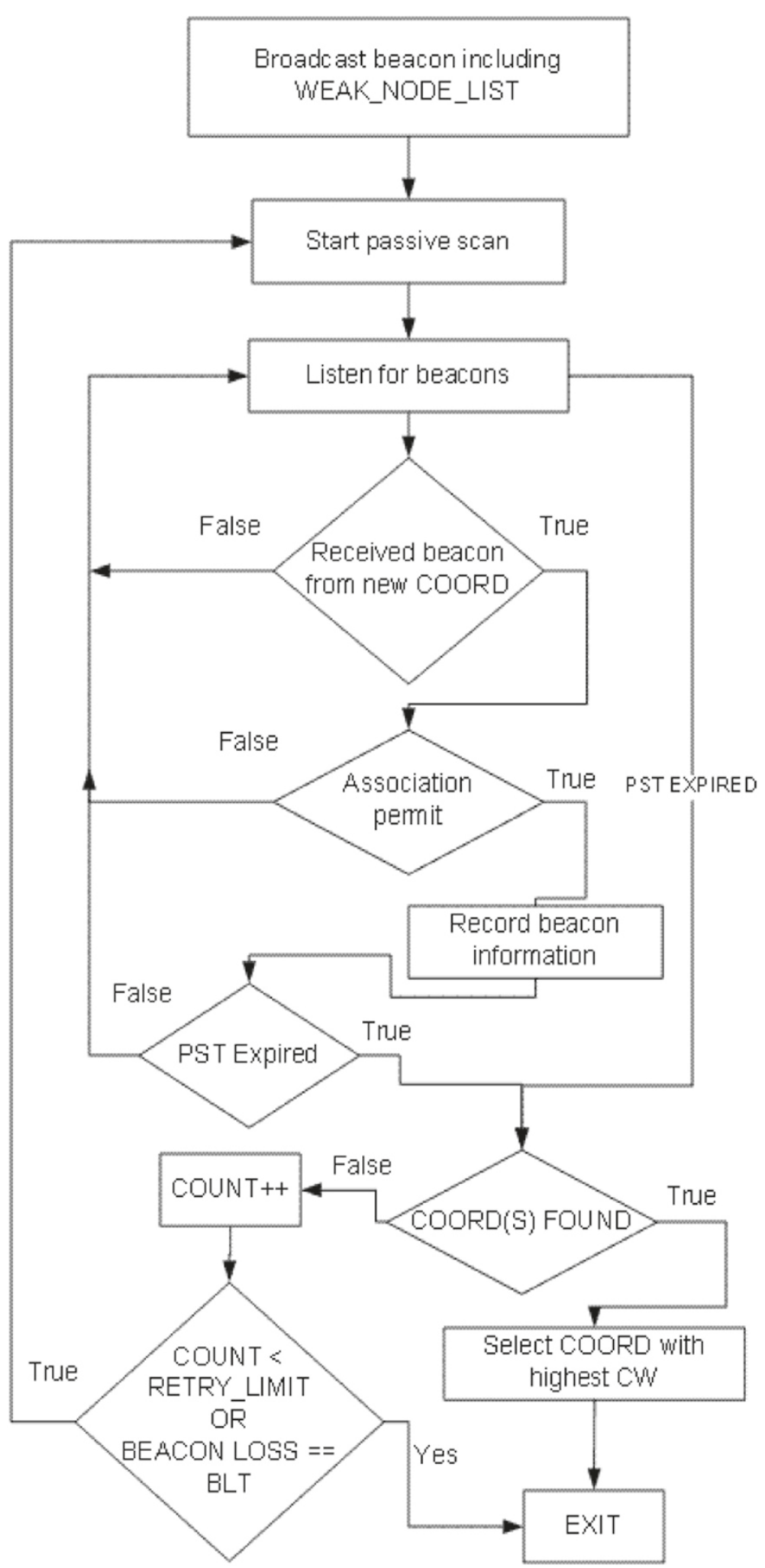

Figure 4 Flow chart: preemptive re-association decision. 


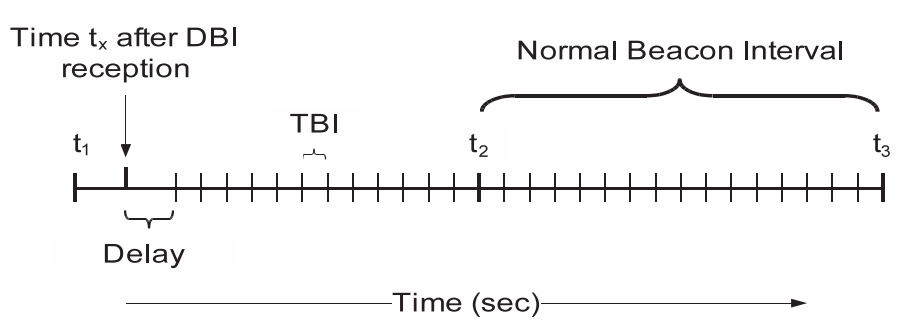

Figure 5 Synchronizing TBI and normal beacon interval.

\subsection{Adjustment of beacon interval length}

The proposed PRA scheme can be useful in high BO networks or in dynamically adjusted BO networks. However, if $\mathrm{BO}$ is high, coordinator discovery during RAP is difficult to achieve even with low speed of mobile node. This is because of longer beacon interval of coordinators. Therefore, in high BO WPANs, swift coordinator discovery is only possible if the $\mathrm{BO}$ of neighboring coordinators is decreased.

$\mathrm{BO}$ reduction at neighboring coordinators is performed prior to the arrival of mobile node in their cluster. In PRA, the parent coordinator broadcasts this BO information using decrease beacon interval (DBI) command message [15]. DBI message is transmitted by the coordinator because of the following reasons. Firstly, in WPANs, RFDs have limited energy resources and conserving energy is the most important objective. Therefore, coordinator (FFD) transmits DBI message instead of RFD. Secondly, in beacon-enabled IEEE 802.15.4 networks, during reassociation, RFDs discover the network using passive scan and do not explicitly request for beacons. Since PRA complements and optimizes the performance of beacon-enabled IEEE 802.15.4, transmission of DBI messages by RFDs is not appropriate. Lastly, the coordinator performs PRD; thus, it transmits the DBI command message.

The DBI message is transmitted to all one-hop coordinators by the parent coordinator during PRD, when a node is added in weak node list. The DBI command message instructs the neighboring coordinator(s) to decrease their beacon interval to the ideal beacon order (IBO). IBO is defined by the application depending on its data rate. The coordinator receiving DBI message can decrease its $\mathrm{BO}$ immediately or after expiration of current beacon interval. If $\mathrm{BO}$ is immediately decreased, the member nodes can lose synchronization with coordinator. On the other hand, decreasing $\mathrm{BO}$ after the completion of current beacon interval can considerably increase the passive discovery time. Therefore, coordinators issue temporary beacons after receiving DBI message. The length of temporary beacon interval (TBI) corresponds to IBO. These beacons are only used by non-member nodes for new associations. TBI length is an offset of the remaining time in the current beacon interval. Consider $t_{1}$ and $t_{2}$ shown in Figure 5 as successive beacon transmission times by the coordinator. In this case, the coordinator receives DBI message during current beacon interval $t_{1}, t_{2}$, and decides to issue temporary beacons at time $t_{x}$. Then, the initial delay before the

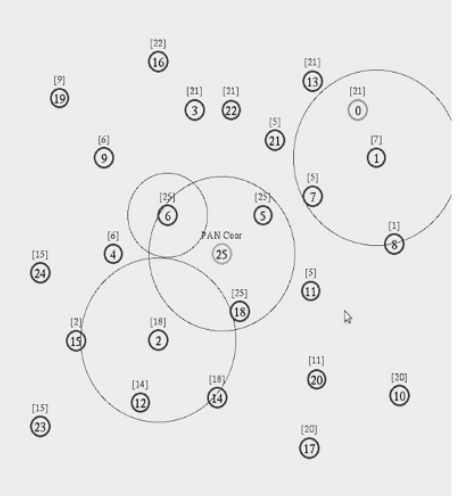

(a) 25 FFDs and 1 RFD

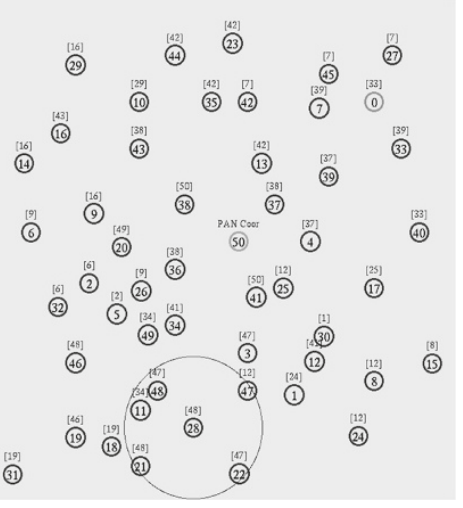

(b) 50 FFDs and 1 RFD

Figure $\mathbf{6}$ Simulation setup in $\mathbf{5 0} \times \mathbf{5 0} \mathbf{m}$ field. (a) 25 FFDs and 1 RFD. (b) 50 FFDs and 1 RFD. 
Table 1 Network parameters

\begin{tabular}{lc}
\hline Parameters & Unit or type \\
\hline Traffic & CBR \\
Packet size & $50 \mathrm{~B}$ \\
Network layer & AODV \\
Radio range & $10 \mathrm{~m}$ \\
Frequency band & $2.4 \mathrm{GHz}$ \\
Wireless channels & 3 \\
Buffer size & 10 packets \\
Data rate & $10 \mathrm{Kbps}$ \\
Min LQI_THRESHOLD & 150 \\
WAIT_LIMIT & 3 \\
IBO & 4 \\
Device speeds & $0.5 \mathrm{and} 1 \mathrm{~m} / \mathrm{s}$ \\
Initial node energy & $4 \mathrm{Joules}$ \\
Transmit power & $46.2 \mathrm{~mW}$ \\
Receive power & $41.3 \mathrm{~mW}$ \\
Idle & $0.003 \mathrm{~mW}$ \\
\hline
\end{tabular}

broadcast of first temporary beacon by the coordinator is calculated by using (2).

$$
\text { Delay }=\left(t_{2}-t_{x}\right) \% \mathrm{TBI}
$$

The temporary beacon transmission is only started if $\mathrm{BO}$ is greater than $\mathrm{IBO}$ at the time of DBI reception. These beacons are issued only for short duration corresponding to passive scan durations according to IBO.

\section{Performance analysis}

In this section, detailed performance evaluation of PRA is presented based on simulation analysis of network simulator Ns-2 [18]. Comparison of PRA is carried out against re-association mechanism of IEEE 802.15.4 and Enh-802.15.4 [9]. In simple IEEE 802.15.4, the association request is sent after passive scan to the first discovered coordinator having LQI above threshold (127). On the other hand, in Enh-802.15.4 [9], a coordinator having the highest LQI and the smallest timestamp value is selected. PRA uses LQI threshold of 150 based on the findings in [15], where authors show the decrease in LQI values with distance of mobile node from the coordinator. It is observed that LQI values suddenly start to decrease once the device comes near the edge of coverage range of coordinator.

Simulation setup is shown in Figure 6 that comprises of beacon-enabled WPANs randomly deployed in $50 \times 50$ $\mathrm{m}$ field. The network comprises of a single PAN coordinator, a RFD, and FFDs. In this section, we use mobile cluster head (mobile- $\mathrm{CH}$ ) terminology to refer to moving FFD or coordinator whereas mobile device refers to RFD or end device. Mobile- $\mathrm{CH}$ and mobile device continuously moves within the network and follow a random mobility model with speeds of 0.5 or $1 \mathrm{~m} / \mathrm{s}$. All the cluster heads transmit beacon frames, and $\mathrm{BO}$ is initially set to 4 . In all simulations, $\mathrm{SO}$ is same as BO. The mobile device transmits data to the PAN coordinator using multi-hop data forwarding. Data are collected after network is established and all cluster heads are successfully transmitting their beacons. Data reporting period in all simulations is 500 $\mathrm{s}$ and averaged results after 10 simulation runs are presented. Results are taken from the same simulation setup but on different node speeds therefore confidence level

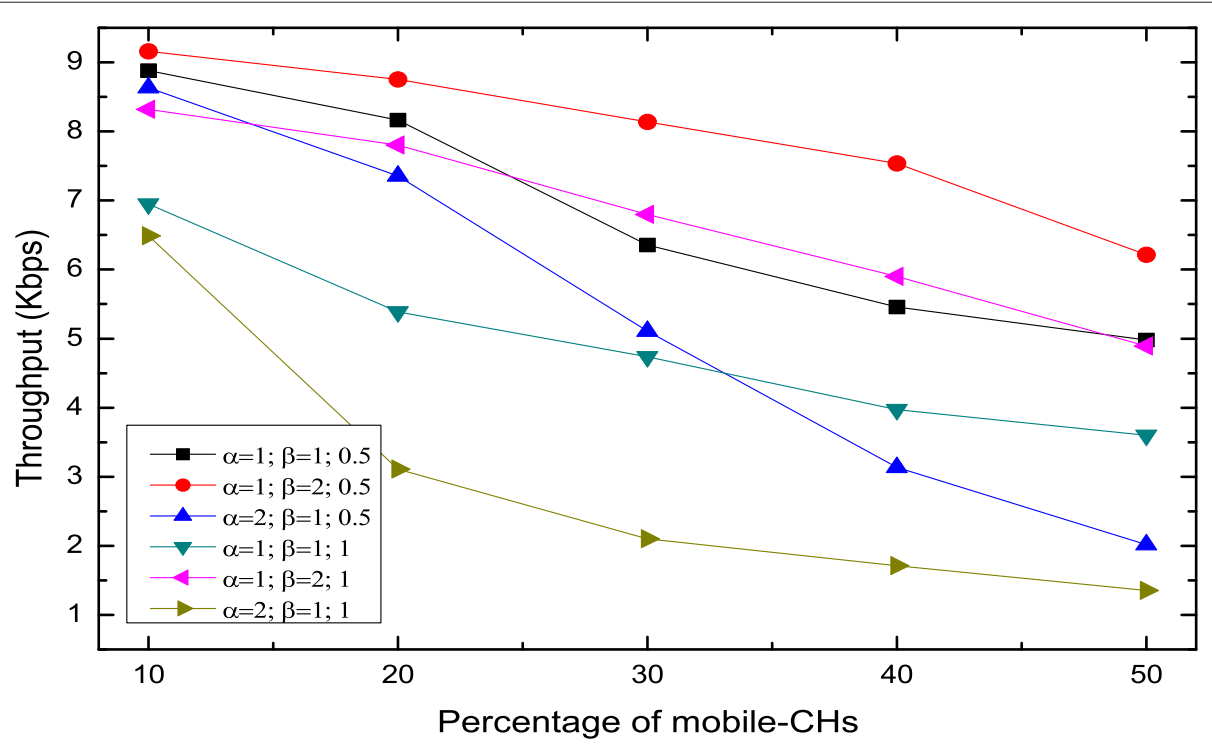

Figure 7 Throughput of PRA at 0.5 and $1 \mathrm{~m} / \mathrm{s}$ using different LQI and mobility weights. 


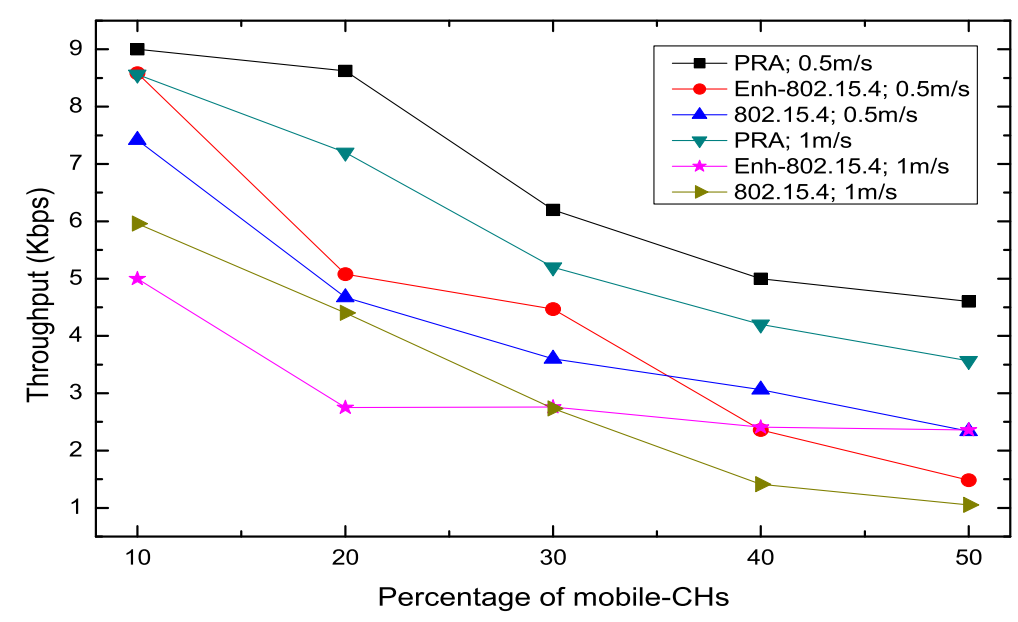

Figure 8 Throughput observed by varying number of mobile-CHs in a 25-node network.

observed for PRA results is $96 \%$ and $85 \%$ at 0.5 and $1 \mathrm{~m} / \mathrm{s}$, respectively. Lower confidence level at higher node speed is because when a mobile node moves away from the parent coordinator sometimes it is not properly connected to next coordinator. Although, in all such cases, it selects a next coordinator but parent coordinators connectivity in the clustered tree topology is lost. As a result, data delivery stops and lower throughput is observed. This situation occurs in clustered tree topology as sometimes the nodes are unable to receive beacons from their parent coordinators in high mobility scenarios. Network parameters are listed in Table 1.

Performance of PRA depends on proper selection of link quality and MF weights. MF can be given less, equal, and more preference than LQI during coordinator selection. Therefore, we have used three test cases with $\alpha=$ $2 \beta=1, \alpha=1 \beta=1$, and $\alpha=1 \beta=2$, representing lesser, equal, and higher preference of MF than LQI, respectively. Throughput observed at the PAN coordinator during $500 \mathrm{~s}$ of data reporting by the mobile device is shown in Figure 7. At lower mobility level with $10 \%$ to $20 \%$ of mobile-CHs, higher throughput is observed if MF has equal or higher weight than LQI. However, at higher mobility level with $30 \%$ to $50 \%$ of mobile-CHs or at faster speeds of $1 \mathrm{~m} / \mathrm{s}$, assigning more weight to MF gives better throughput. This is because the mobile device attempts to select static cluster heads with stable links. Therefore, PRA uses $\alpha=1$ and $\beta=2$ as LQI and MF weights in mobile environments.

In the remaining of this section, performance of PRA and IEEE 802.15.4 is shown for throughput, connectivity time, and re-association latency.

\subsection{Throughput}

Observed throughput at the PAN coordinator in 25 and 50 nodes WPAN are shown in Figures 8 and 9, respectively.

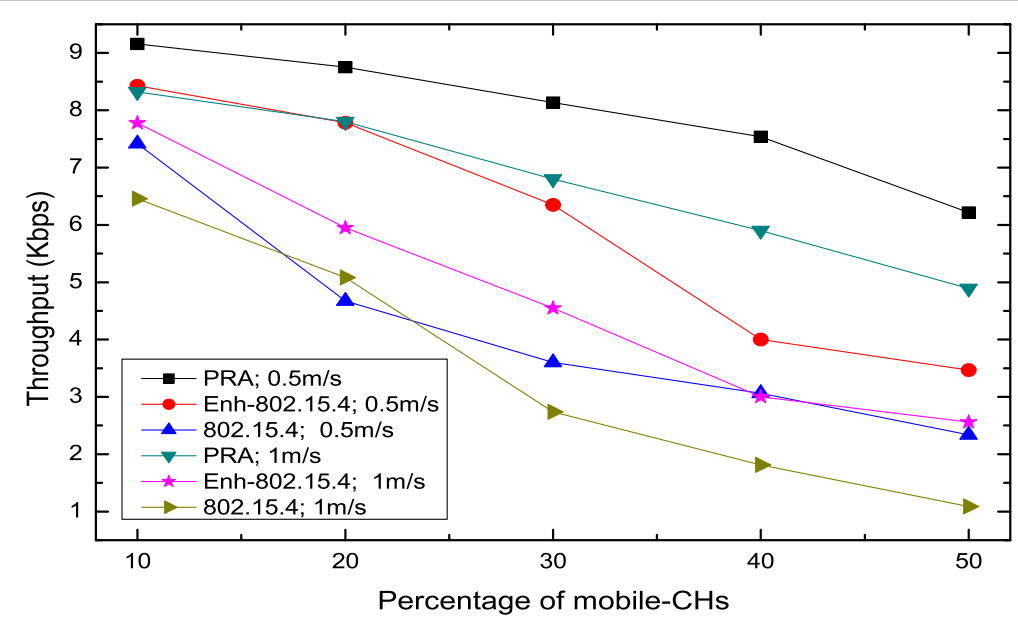

Figure 9 Throughput observed by varying number of mobile-CHs in a 50-node network. 


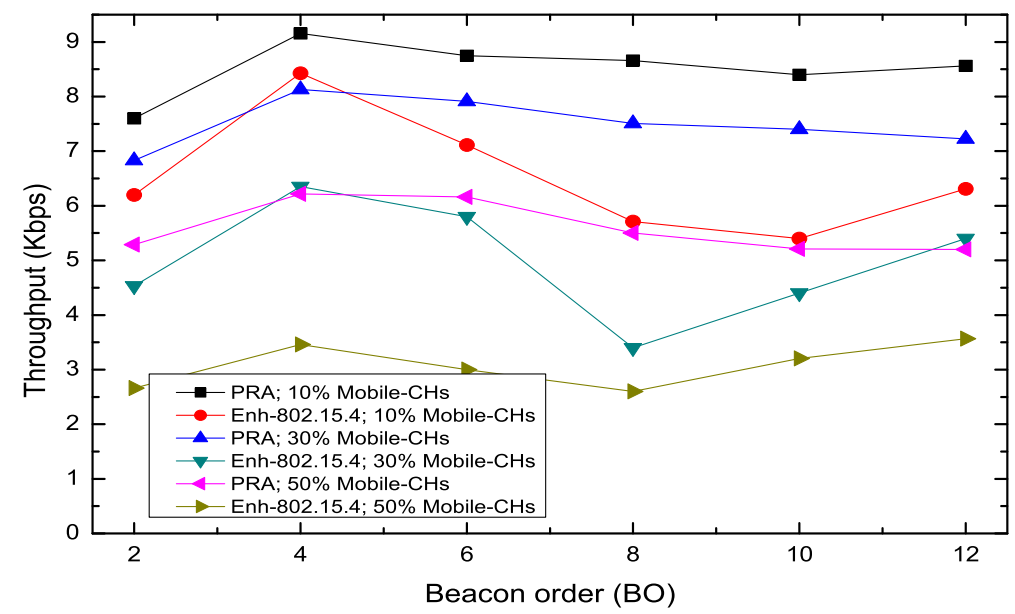

Figure 10 Throughput observed by varying $\mathrm{BO}$ and number of mobile-CHs at speed of $0.5 \mathrm{~m} / \mathrm{s}$.

PRA outperforms both IEEE 802.15.4 and enhanced IEEE 802.15.4 [9] with respect to throughput at different levels of mobility. With fewer nodes in the network, mobility severely degrades the throughput. This is evident by lower throughput observed by all schemes in Figure 8 as compared to Figure 9. Also, the higher the speed of devices within the network, the lower is the throughput. This is because connectivity is frequently lost and data forwarding requires re-establishment of path after every re-association. However, in PRA, the throughput is significantly higher than other schemes in Figures 8 and 9 because mobile device attempts to attach with lesser mobile-CHs. Also, PRA has shorter re-association latency which contributes to swift re-association, resulting in quick re-establishment of source to destination path.

Observed throughput by varying $\mathrm{BO}$ of the network in a 50-node WPAN are shown in Figures 10 and 11 for device speeds of 0.5 and $1 \mathrm{~m} / \mathrm{s}$, respectively. PRA provides consistently higher throughput at all $\mathrm{BO}$ than IEEE 802.15.4 and enhanced IEEE 802.15.4 [9]. This is because

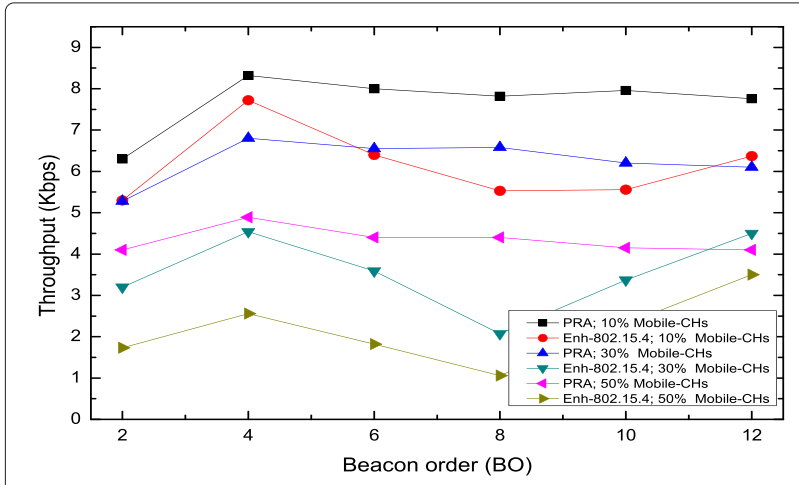

Figure 11 Throughput observed by varying $\mathrm{BO}$ and number of mobile-CHs at speed of $1 \mathrm{~m} / \mathrm{s}$.
PRA decreases the passive discovery time in high $\mathrm{BO}$ networks by lowering the $\mathrm{BO}$ of coordinators near the mobile device. On the other hand, throughput using both variants of IEEE 802.15.4 decreases with the increase in BO. But at $\mathrm{BO}=12$ or higher, throughput slightly increases because mobile device continues to transmit data even if it moves out of mobile- $\mathrm{CH}$ coverage area. Since cluster change is detected after loss of successive beacons, it takes large time to detect loss of connectivity at high BO.

\subsection{Coordinator connectivity time}

Coordinator connectivity time is a good measure for estimating the frequency of re-association. The higher the connectivity time of devices with coordinator, the lesser is the frequency of re-association and vice versa. Average connectivity time per coordinator for mobile device in 50-node network with increasing number of mobile$\mathrm{CHs}$ is shown in Figure 12. PRA shows increased percoordinator connectivity time as compared to enhanced IEEE-802.15.4 that selects coordinator with highest LQI

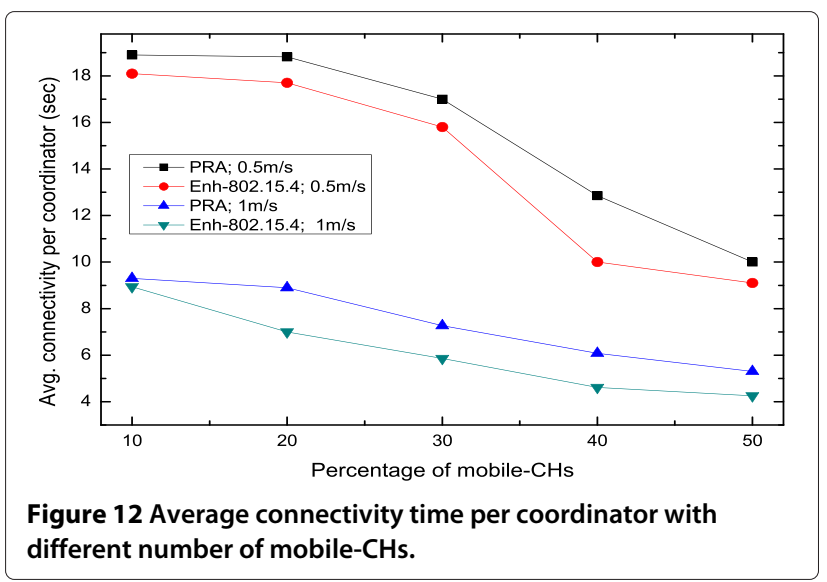




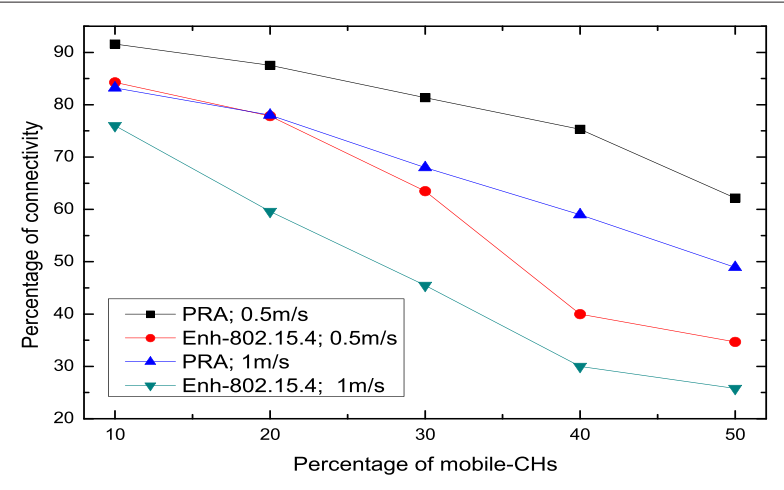

Figure 13 Percentage of end-to-end data connectivity time between the mobile device and the PAN coordinator.

during re-association. On the other hand, connectivity of mobile device with coordinators is increased by PRA as it selects lesser mobile coordinators having good link quality during re-association. In order to further validate this fact, the percentage of end-to-end data connectivity time between the mobile device and the PAN coordinator, during 500s of data reporting time, is shown in Figure 13. If there is an active data forwarding path from source to destination, end-to-end connectivity is available. PRA increases the end-to-end connectivity as re-association frequency is lesser in PRA as compared to enhanced IEEE 802.15.4 [9].

\subsection{Re-association latency}

The time spent by mobile device on coordinator discovery and exchange of messages during re-association is collectively referred as re-association latency. Average time spent on re-association by mobile device in a 50-node network with increasing number of mobile- $\mathrm{CHs}$ is shown in Figure 14. Average re-association latency of PRA is significantly less than the enhanced IEEE 802.15.4 because it does not perform orphan scan and passive scan is performed only on current channel. At higher mobility level,

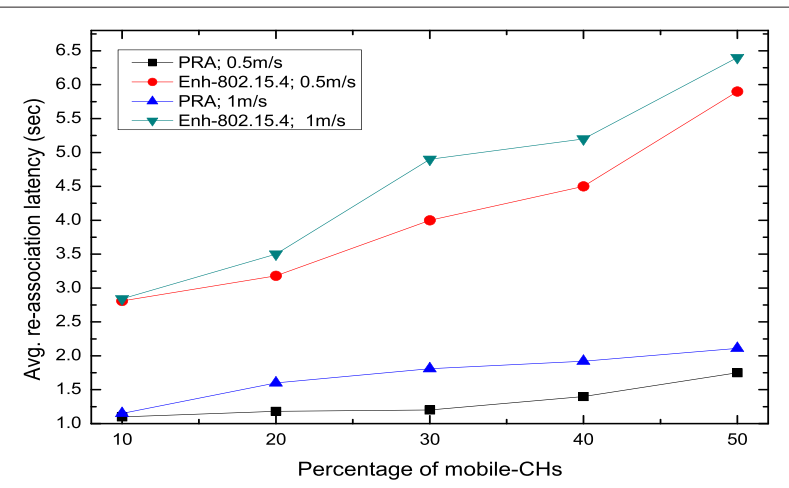

Figure 14 Average time spent on each re-association with different number of mobile-CHs.

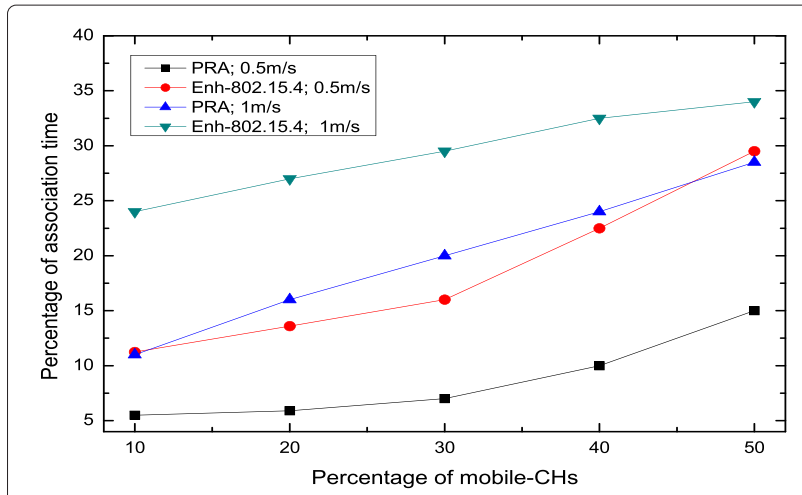

Figure 15 Percentage of time that the mobile device spends on re-associations during $500 \mathrm{~s}$ of simulation.

when a mobile device is performing lengthy re-association procedure of IEEE 802.15.4 with cluster head, the devices move out of each other's communication range due to mobility. As a result, scanning procedure is repeated to find new coordinator, thus increasing the latency of reassociation. This fact is further evident in Figure 15 that shows the percentage of time spent by the end device on re-associations during $500 \mathrm{~s}$ of simulation. Although PRA spends sizeable time at $1 \mathrm{~m} / \mathrm{s}$ on re-association, but average re-association of PRA shown in Figure 14 is sufficiently less than enhanced IEEE 802.15.4 [9]. This is because of greater number of successful re-associations at higher node speed.

\subsection{Energy consumption}

Total energy consumed within the network using PRA, Enh-IEEE 802.15.4, and PRA+BOA (beacon order adaption) with one PAN coordinator, 50 FFD and one source node in random topology is shown in Figure 16. PRA+BOA uses beacon order adaption scheme that is similar to [3] and selects $B O$ between 4 and 12, respectively. After $100 \mathrm{~s}$, the mobile node starts transmitting

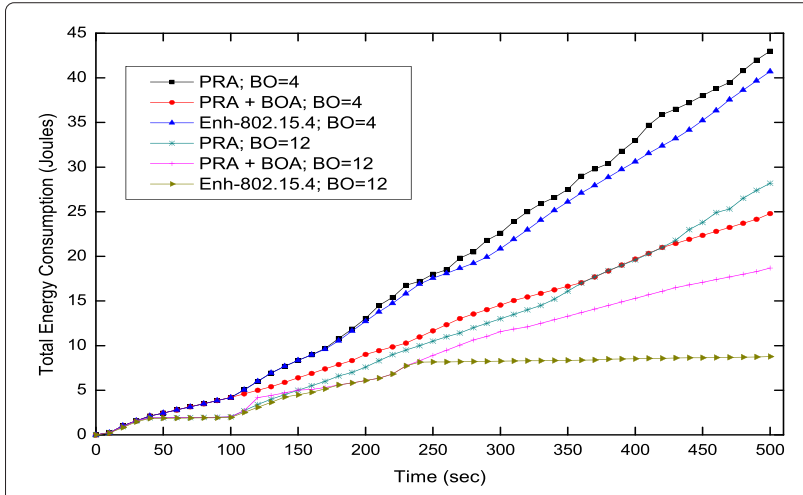

Figure 16 Network energy consumption during 500s of simulation. 
data to the PAN coordinator. At lower $\mathrm{BO}$ and without BO scheduling, energy is consumed because of both beacon transmissions and data delivery. As a result, continuous increase in energy consumption is observed at lower BO (4) for both Enh-IEEE 802.15.4 and PRA. At higher $\mathrm{BO}$, since beacon frequency is very low, therefore the energy of nodes is mostly utilized because of data delivery. Enh-IEEE 802.15.4 at higher BO (12) has lower energy consumption that too becomes negligible after around $240 \mathrm{~s}$ in the simulation time. This is because when the mobile device moves away from the parent coordinator, it is unable to make new association. As a result, the device does not transmit data and network energy is not consumed significantly. Energy utilization of PRA improves by using $\mathrm{BOA}$ scheme, as it increases the $\mathrm{BO}$ of clusters not involved in communication.

\section{Conclusions}

In this paper, we have presented a PRA scheme for mobile WPAN. Simulation results have shown that PRA successfully decreases both latency and frequency of reassociation for WPAN devices. The use of both LQI and MF of coordinator helps in selecting links that can provide longer connectivity to reduce re-association frequency. In our simulation study, PRA provided from 5\% to $30 \%$ more connectivity per coordinator than IEEE 802.15.4. On the other hand, swift re-association procedure of PRA reduces the latency of re-association 2 to 3.5 times as compared to IEEE 802.15.4. PRA is especially useful because it does not contradict with re-association process of IEEE 802.15.4. It is triggered before the actual loss of connectivity is detected by the devices. Therefore, even if swift re-association attempt is unsuccessful using PRA, re-association using IEEE 802.15.4 can be carried out.

Competing interests

The authors declare that they have no competing interests.

\section{Acknowledgement}

This study was supported by research funds from Chosun University, 2014.

\section{Author details}

${ }^{1}$ Department of Computer Science, Bahria University, Islamabad 44000, Pakistan. ${ }^{2}$ Department of Information and Communication Engineering, Chosun University, Gwangju 501-759, South Korea.

Received: 8 August 2013 Accepted: 29 May 2014

Published: 17 June 2014

\section{References}

1. F Cuomo, S Della Luna, E Cipollone, P Todorova, T Suihko, Topology formation in IEEE 802.15.4: cluster-tree characterization, in Proc. IEEE PerCom 08, (2008), pp. 276-281

2. R Szewczyk, A Mainwaring, J Polastre, J Anderson, D Culler, An analysis of a large scale habitat monitoring application, in Proc. ACM SenSys'04, (2004), pp. 214-226

3. A Soomro, D Cavalcanti, Opportunities and challenges in using WPAN and WLAN technologies in medical environments. [from Open Call] IEEE Comm. Mag. 45(2), 114-122 (2007)
4. YC Tseng, MS Pan, YY Tsai, Wireless sensor networks for emergency navigation. Comput. J. 39(7), 55-62 (2006)

5. H Yang, SH Yang, 'Connectionless indoor inventory tracking in Zigbee RFID sensor network, in Proc. IEEE IECON'09, (2009), pp. 2618-2623

6. LJ Chen, T Sun, NC Liang, An evaluation study of mobility support in zigbee networks. J. Signal Process. Syst. 59(1), 111-122 (2010)

7. F Silva, C Branquinho, M Assumpcao, Mobility impact on IEEE 802.15.4 network through a simulation platform. IEEE Latin Am. Trans. 9(5), 655-652 (2011)

8. C Chaabane, C Pegatoquet, M Auguin, M Jemaa, Energy optimization for mobile nodes in a cluster tree IEEE 802.15.4/ZigBee network, in Proc. IEEE ComComAp'12, (2012), pp. 328-333

9. K Zen, D Habibi, I Ahmad, Improving mobile sensor connectivity time in the IEEE 802.15.4 networks, in Proc. IEEE ANTAC'08, (2008), pp. 317-320

10. A Willig, N Karowski, J Hauer, Passive discovery of IEEE 802.15.4-based body sensor networks. J. Ad Hoc Netw. 8(7), 742-754 (2010)

11. P Dutta, D Culler, Practical asynchronous neighbor discovery and rendezvous for mobile sensing applications, in Proc. ACM SenSys'08, (2008), pp. 71-84

12. Y $\mathrm{Li}, \mathrm{Y}$ Pan, $\mathrm{P}$ Wang, Research and implementation of a mobility management mechanism for Wireless Sensor Networks based on IEEE 802.15.4, in Proc. IEEE CYBER'11, (2011), pp. 260-264

13. F Zhang, F Wang, B Dai, Y Li, Performance evaluation of IEEE 802.15.4 beacon-enabled association process, in Proc. IEEE AINAW'08, (2008), pp. 541-546

14. E Meng, Y Han, A new association scheme of IEEE 802.15.4 for real-time applications, in IEEE WiCom'09, (2009), pp. 1-5

15. F Bashir, WS Baek, P Sthapit, D Pandey, JY Pyun, Coordinator assisted passive discovery for mobile end devices in IEEE 802.15.4, in Proc. of IEEE CCNC'13 Work-in-Progress, (2013), pp. 586-589

16. R Alberola, D Pesch, Duty cycle learning algorithm (DCLA) for IEEE 802.15.4 beacon-enabled wireless sensor networks. Ad Hoc Net. J. 10(4), 664-679 (2012)

17. J Lim, B Jang, Dynamic duty cycle adaptation to real-time data in IEEE 802.15.4 based WSN, in Proc. IEEE CCNC'08, (2008), pp. 353-357

18. Ns2. http://nsnam.isi.edu/nsnam/index.php/Main_Page

\section{doi:10.1186/1687-1499-2014-97}

Cite this article as: Hussain and Pyun: Preemptive re-association scheme for beacon-enabled wireless personal area networks. EURASIP Journal on Wireless Communications and Networking 2014 2014:97.

\section{Submit your manuscript to a SpringerOpen ${ }^{\mathcal{O}}$ journal and benefit from: \\ - Convenient online submission \\ - Rigorous peer review \\ - Immediate publication on acceptance \\ - Open access: articles freely available online \\ - High visibility within the field \\ - Retaining the copyright to your article}

Submit your next manuscript at $>$ springeropen.com 